\title{
A novel PNPLA6 compound heterozygous mutation identified in a Chinese patient with Boucher-Neuhäuser syndrome
}

\author{
RUIZHI ZHENG ${ }^{1 *}$, YAGUANG ZHAO ${ }^{2 *}$, JIAYU WU $^{2 *}$, YUANMEI WANG $^{2}$, JIAN-LING LIU $^{3}$, \\ ZHI-LING ZHOU ${ }^{3}$, XIAO-TAO ZHOU ${ }^{4}$, DAN-NA CHEN ${ }^{5}$, WEI-HUA LIAO ${ }^{3}$ and JIA-DA LI ${ }^{2}$ \\ ${ }^{1}$ Department of Endocrinology, The People's Hospital of Henan Province, Zhengzhou, Henan 450003; \\ ${ }^{2}$ Center for Medical Genetics, School of Life Sciences, Central South University, Changsha, Hunan 410078; \\ ${ }^{3}$ Department of Radiology, Xiangya Hospital, Central South University, Changsha, Hunan 410083; \\ ${ }^{4}$ Department of Immunology, Xinjiang Medical University, Urumqi, Xinjiang Uygur Autonomous Region 830054; \\ ${ }^{5}$ Department of Basic Medical Sciences, Changsha Medical University, Changsha, Hunan 410219, P.R. China
}

Received October 28,2017; Accepted March 20, 2018

DOI: $10.3892 / \mathrm{mmr} .2018 .8955$

\begin{abstract}
The combination of cerebellar degeneration, hypogonadotropic hypogonadism and chorioretinal dystrophy defines Boucher-Neuhäuser syndrome (BNS), which has been associated with autosomal-recessive mutations in the patatin-like phospholipase domain containing 6 (PNPLA6) gene. However, no BNS cases have been reported in mainland China. In the present study, to the best of the authors' knowledge, the first patient with BNS was identified in China. A 39-year-old male was first diagnosed with hypogonadotropic hypogonadism. The proband additionally exhibited retinal degeneration and cerebellar dystrophy. Whole exome sequencing identified a compound heterozygous mutation in PNPLA6 (c.3386G $>\mathrm{T}+$ c. $3534 \mathrm{G}>\mathrm{C}$ ). The mutant amino acids were highly conserved and the mutations were predicted to be deleterious. This result further confirmed the role of PNPLA6 in BNS and suggested that whole exome sequencing may be applied for the diagnosis of complex syndromes, including BNS, prior to the observation of obvious symptoms.
\end{abstract}

Correspondence to: Professor Jia-Da Li, Center for Medical Genetics, School of Life Sciences, Central South University, 110 Xiangya Road, Changsha, Hunan 410078, P.R. China

E-mail: lijiada@sklmg.edu.cn

Dr Wei-Hua Liao, Department of Radiology, Xiangya Hospital, Central South University, 87 Xiangya Road, Changsha, Hunan 410083, P.R. China

E-mail: ouwenliao@163.com

*Contributed equally

Key words: Boucher-Neuhäuser syndrome, patatin-like phospholipase domain containing 6 , whole exome sequencing, compound heterozygous mutation

\section{Introduction}

Boucher-Neuhäuser syndrome [BNS; Mendelian Inheritance in Man (MIM) reference no. 215470; www.omim.org] is a rare autosomal recessive disorder and is characterized by spinocerebellar ataxia, eye abnormalities and a failure of the pituitary gland to stimulate gonadal development during puberty (1-8). Previously, mutations in patatin-like phospholipase domain containing 6 (PNPLA6) have been linked to BNS (9-13). PNPLA6 (MIM reference no. 603197; www.omim.org; 19p13.2) encodes for a neuropathy target esterase $(13,14)$. A total of five different transcripts have been identified, with the longest transcript, transcript variant 1 (GenBank accession no. NM_001166111.1; www.ncbi. nlm.nih.gov/genbank), encoding a protein (GenBank accession no. NP_001159583.1) of 1,375 amino acids (14). In addition to BNS, variations in the PNPLA6 gene have been implicated in Gordon Holmes syndrome, spastic paraplegia, photoreceptor degeneration and pure cerebellar ataxia (12,15-17).

Hypogonadotropic hypogonadism may be identified at the onset of puberty (18-20). Gait ataxia in BNS generally manifests prior to early adulthood, although late-onset ataxia has additionally been reported $(2,9)$. In the present study, a 39-year-old male was initially diagnosed with hypogonadotropic hypogonadism at the age of 14 years. Subsequent magnetic resonance imaging (MRI) and funduscopic examination suggested that the symptoms were a result of BNS. The cause of the symptoms was further confirmed by the presence of a compound heterozygous PNPLA6 mutation.

\section{Materials and methods}

Patients. The proband and his parents were recruited at the People's Hospital of Henan Province (Zhengzhou, China) in March 2016. The age range of patients was 39-70 years old. Written informed consent was obtained. The present study was approved by the ethics committees of Central South University and The People's Hospital of Henan Province.

Clinical assessments. Endocrine manifestation in serum isolated from whole blood samples $(1 \mathrm{ml}$ per patient) were 
examined using the following ELISA kits: Human luteinizing hormone ELISA kit (cat. no. ab108651; Abcam, Cambridge, UK), human follicle stimulating hormone ELISA kit (cat. no. ab108641; Abcam), human estradiol E2 ELISA kit (cat. no. ab108640; Abcam), human testosterone ELISA kit (cat. no. ab174569; Abcam), human prolactin ELISA kit (cat. no. ab108655; Abcam), human progesterone ELISA kit (cat. no. ab108654; Abcam), human adrenocorticotropic hormone ELISA kit (cat. no. ENZ-KIT138-0001; Enzo Life Sciences, Inc., Farmingdale, NY, USA), human triiodothyronine ELISA kit (cat. no. ab108664; Abcam), human thyroxine ELISA kit (cat. no. ab108686; Abcam), human thyroid stimulating hormone ELISA kit (cat. no. ab108659; Abcam) and human parathyroid hormone ELISA kit (cat. no. ab230931; Abcam). The retina was examined using routine funduscopic examination; the reflexes of the two legs were detected using electromyography; and the brain structure was examined using MRI. The results of MRI were analyzed using fluid-attenuated inversion recovery (21). And the middle cerebral artery was examined using magnetic resonance angiography (22).

Whole exome sequencing. Genomic DNA was isolated from peripheral blood leukocytes ( $1 \mathrm{ml}$ per patient). The whole exome was captured using a Sure Select Human All Exon kit (Agilent Technologies, Inc., Santa Clara, CA, USA), and was sequenced on an Illumina HiSeq2500 instrument (Illumina, Inc., San Diego, CA, USA). The sequenced reads were aligned to the human genome reference (University of California Santa Cruz hg 19 version; www.epigenomebrowser.org/index.html) using the Burrows-Wheeler Aligner (bwa-0.7.17) (23). Read qualities were recalibrated using the Genome Analysis Toolkit (GATK-4.0.2.1; Broad Institute, Cambridge, MA, USA). The GATK IndelRealigner was used to realign reads around insertion/deletion sites. The single nucleotide variants (SNVs) and small insertions and deletions (InDels) were generated with the GATK Unified Genotyper in parallel with the SAMtools (samtools-1.7; www.htslib.org/doc/samtools.html) pipeline. ANNOVAR (version 2.3.1) (24) was used to annotate the detected variations. ExAC (version 0.3.1; exac.broadinstitute.org) and 1,000 Genomes (www.internationalgenome.org) (25) were used to identify the population frequencies of mutations.

Deleterious missense SNVs were predicted using the following tools: i) SIFT [sift.jcvi.org; a single nucleotide polymorphism (SNP) with SIFT score $<0.05$ predicts a negative effect on the encoded amino acid]; ii) Polyphen2 (genetics. bwh.harvard.edu/pph2; a SNP with score between 0.85-1.0 may be predicted to be damaging to the encoded amino acid, while a SNP with score of 0.0-0.15 may be predicted to be benign, and a SNP with score of $0.15-1.0$ predicts possible damage); iii) MutationTaster (www.mutationtaster.org; a score close to 1 indicates the given variant to be disease-causing); iv) MutationAssessor (mutationassessor.org/r3; High/Medium means functional and Low/Neutral means non-functional); and v) Combined Annotation Dependent Depletion (CADD; cadd.gs.washington.edu), in which a scaled CADD score of 20 means that a variant is among the top $1 \%$ of deleterious variants in the human genome, and a scaled CADD score of 30 means that the variant is in the top $0.1 \%$ (26).
Polymerase chain reaction (PCR)-sanger sequencing. PCR was used to amplify the exons containing the PNPLA6 variants identified in the whole exome sequencing. PCR was performed in a total volume of $20 \mu \mathrm{l}$ with $50 \mathrm{ng}$ genomic DNA as template, $10 \mu 1$ X Taq Mastermix (E005; Novoprotein Scientific, Inc., Summit, NJ, USA), $1 \mu \mathrm{l}$ forward primer $(10 \mu \mathrm{mol} / \mathrm{ml})$ and $1 \mu \mathrm{l}$ reverse primer $(10 \mu \mathrm{mol} / \mathrm{ml})$ under the following thermocycling conditions: $94^{\circ} \mathrm{C}$ for $2 \mathrm{~min}$; followed by 40 cycles of $94^{\circ} \mathrm{C}$ for $20 \mathrm{sec}, 65^{\circ} \mathrm{C}$ for $20 \mathrm{sec}$ and $72^{\circ} \mathrm{C}$ for $45 \mathrm{sec}$; and a final extension step at $72^{\circ} \mathrm{C}$ for $5 \mathrm{~min}$. The amplified products were used for Sanger sequencing as performed by BioSune Biotechnology Co. (Shanghai, China). The sequencing results were analyzed by using DNA Star-Megalign software (version 3.3.8; DNASTAR, Inc., Madison, WI, USA). The primers used for PCR were as follows: PNPLA6-E29-forward (F), 5'-CAGGCTGTGTGT GGCGTTACGTC-3'; PNPLA6-E29-reverse (R), 5'-GCACAT GTCGCTGTCCACAGGCAC-3'; PNPLA6-E30-F, 5'-GGA CATCGCCCGCAGCATG-3'; and PNPLA6-E30-R, 5'-ATA GATCTGGTCGAACTTCCCAAAG-3'.

Structural modeling of proteins. Structural modeling was performed using I-TASSER (zhanglab.ccmb.med.umich. edu/I-TASSER). The models were visualized using PyMOL (version 0.99; DeLano Scientific LLC., Palo Alto, CA, USA).

\section{Results and Discussion}

Endocrine manifestation. The proband is a 39-year-old male (as of 2016). He was diagnosed with hypogonadotropic hypogonadism 25 years ago at the age of 14 years. He was subsequently treated intermittently for 2 years with testosterone, human chorionic gonadotropin and human menopausal gonadotropin, leading to significant growth in the testes and penis. The proband was married 6 years ago, although he experienced poor sexual performance and the wife of the proband did not conceive. In 2015, the proband was enrolled at The People's Hospital of Henan Province due to infertility.

As illustrated in Table I, the luteinizing hormone (LH), follicle-stimulating hormone (FSH) and testosterone levels of the proband were below normal, whereas the levels of adrenocorticotropic hormone, cortisol and thyroid hormones were in the normal range. A gonadotropin-releasing hormone $(\mathrm{GnRH})$ stimulation test was additionally performed. As exhibited in Table I, GnRH failed to induce an obvious increase in FSH and $\mathrm{LH}$.

Retinal degeneration and cerebellar atrophy. In a routine funduscopic examination, bilateral macular pigment epitheliopathy was identified in the proband (Fig. 1A). Therefore, it was hypothesized that the proband may be affected by BNS.

The proband did not exhibit obvious gait ataxia; however, gait ataxia in patients with BNS may manifest as late as the age of 50 years (9). Therefore a thorough neurological examination was performed. No defect was detected in electroencephalography and hearing tests (data not shown). However, the proband presented with subtle defect in the finger-naseversch and alternate motion tests. In addition, the proband exhibited cavus and a reduced tendon reflex.

Electromyography demonstrated the absence of H-reflection in both legs of the proband (Fig. 1B). MRI demonstrated 
Table I. Baseline hormonal profile and results of gonadotrophin releasing hormone stimulation test.

\begin{tabular}{|c|c|c|c|c|c|c|}
\hline \multirow[b]{2}{*}{ Hormone } & \multicolumn{6}{|c|}{ Time, min } \\
\hline & 0 & 30 & 60 & 90 & 120 & Normal range, basal \\
\hline LH & 0.27 & 0.59 & 0.59 & 0.48 & 0.57 & $1.2-8.6 \mathrm{mIU} / \mathrm{ml}$ \\
\hline FSH & 1.97 & 1.07 & 1.69 & 1.87 & 1.81 & $1.3-19.3 \mathrm{mIU} / \mathrm{ml}$ \\
\hline E2 & 38.32 & & & & & $<53 \mathrm{pg} / \mathrm{ml}$ \\
\hline $\mathrm{T}$ & 2.9 & & & & & $1.75-7.81 \mathrm{ng} / \mathrm{ml}$ \\
\hline PRL & 5.41 & & & & & $2.64-13.13 \mathrm{ng} / \mathrm{ml}$ \\
\hline $\mathrm{P}$ & 0.32 & & & & & $0.1-0.84 \mathrm{ng} / \mathrm{ml}$ \\
\hline \multicolumn{7}{|l|}{ Cortisol } \\
\hline 08.00 a.m. & 8.60 & & & & & $5-25 \mu \mathrm{g} / \mathrm{dl}$ \\
\hline 04.00 p.m. & 5.76 & & & & & $2.5-12.5 \mu \mathrm{g} / \mathrm{dl}$ \\
\hline 12.00 a.m. & $<1.0$ & & & & & $2.9-13 \mu \mathrm{g} / \mathrm{dl}$ \\
\hline \multicolumn{7}{|l|}{ ACTH } \\
\hline 08.00 a.m. & 29.8 & & & & & $12-46 \mathrm{pg} / \mathrm{ml}$ \\
\hline 04.00 p.m. & 22.8 & & & & & $6-23 \mathrm{pg} / \mathrm{ml}$ \\
\hline FT3 & 5.06 & & & & & $3.5-6.5 \mathrm{pmol} / \mathrm{l}$ \\
\hline FT4 & 14.34 & & & & & $11.5-22.7 \mathrm{pmol} / \mathrm{l}$ \\
\hline TSH & 1.217 & & & & & $0.55-4.78 \mu \mathrm{IU} / \mathrm{ml}$ \\
\hline PTH & 71.3 & & & & & $12-88 \mathrm{pg} / \mathrm{ml}$ \\
\hline
\end{tabular}

LH, luteinizing hormone; FSH, follicle-stimulating hormone; E2, estradiol; T, testosterone; PRL, prolactin; P, progesterone; ACTH, adrenocorticotropic hormone; FT3, free triiodothyronine; FT4, free thyroxine; TSH, thyroid stimulating hormone; PTH, parathyroid hormone.
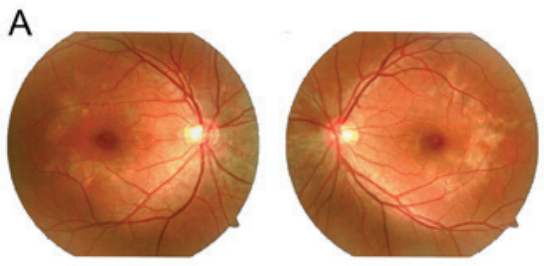

B
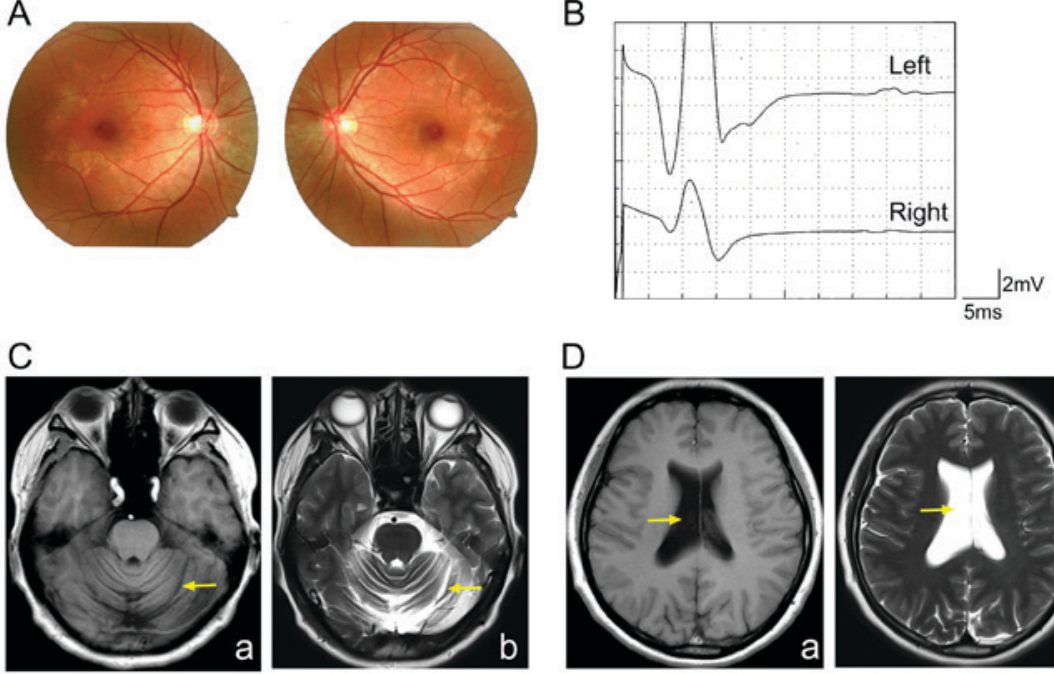

D
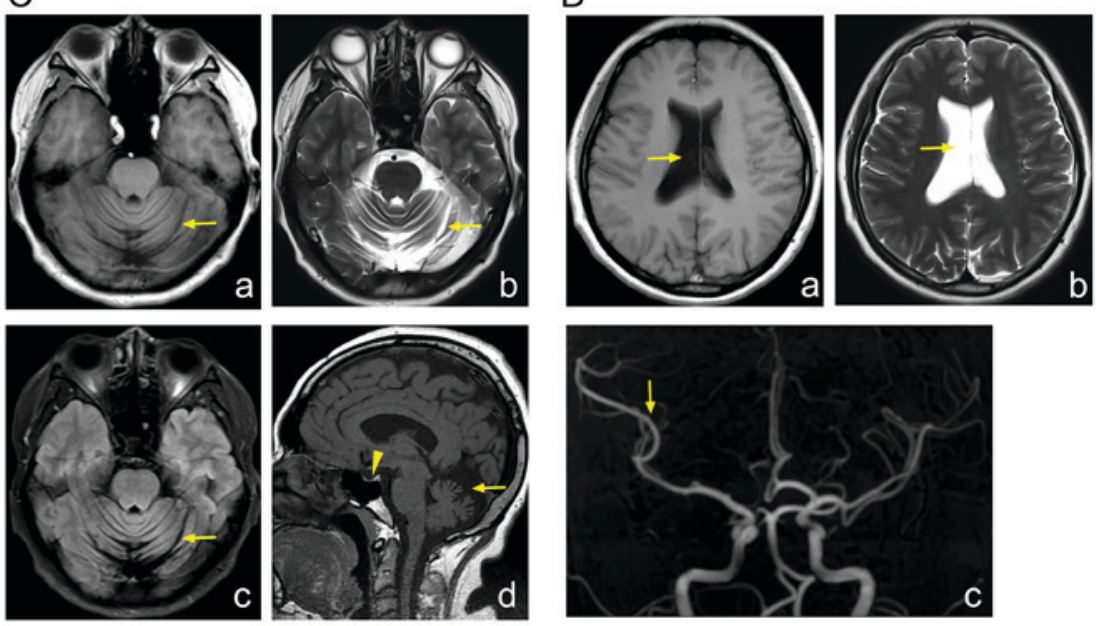

Figure 1. MRI, EMG and funduscopic examination of the proband (II:2). (A) The funduscopic examination demonstrated macular pigment epitheliopathy. (B) EMG illustrated the absence of H-reflection in both legs of the proband. (C) MRI demonstrated marked cerebellar atrophy. The (a) axial T1WI, (b) axial T2WI, and (c) axial fluid-attenuated inversion recovery demonstrated widening of the cerebellar sulci with increased cerebrospinal fluid (indicated by the yellow arrows). (d) Sagittal T1WI demonstrated the widening of cerebellar sulci (indicated by the yellow arrow) and shrinking of pituitary (arrowhead). (D) The axial T1WI (a) and axial T2WI (b) demonstrated the expansion of right lateral side (indicated by the yellow arrows). (c) Magnetic resonance angiography demonstrated a reduction in the branches of right middle cerebral artery (indicated by the yellow arrow). MRI, magnetic resonance imaging; EMG, electromyography. 
A

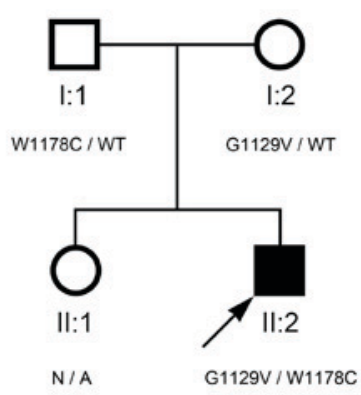

Boucher-Neuhäuser syndrome

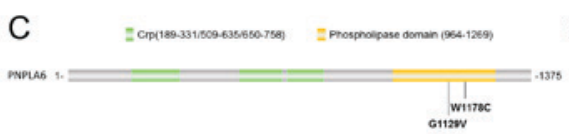

$E$

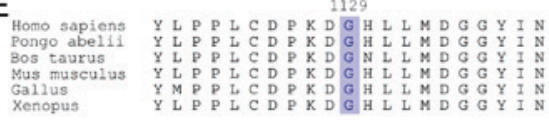

B

II:2

PNPLA6 (c.3386G>T) PNPLA6 (c.3534G>C)
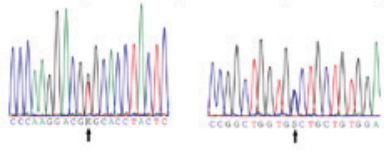

$\mathrm{l}: 1$
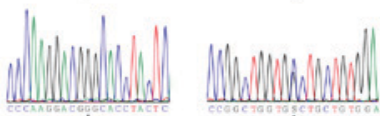

1:2
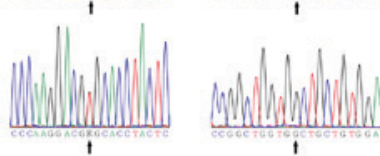

WT
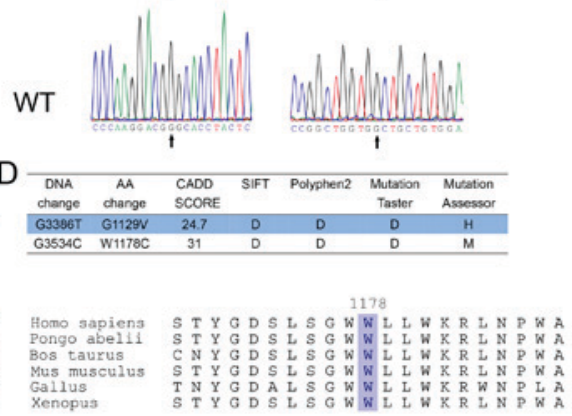

Figure 2. Identification of PNPLA6 mutations in the patient. (A) The pedigree of the family. Subjects in the family are identified by the Roman and Arabic numerals below the symbol, in which the Roman numerals denote the generations. Open symbols, unaffected; filled symbols, affected; squares, male; circles, female; arrow, the proband. (B) Sanger sequencing of codons 3,376-3,396 and 3,524-3,544 of the PNPLA6 genes from the proband (II:2), his father (I:1) and mother (I:2), and a wild-type control. K represents G or T; S represents G or C. (C) Schematic diagram of PNPLA6 protein. The G1129V and W1178C mutations identified in the present study are indicated with arrows. (D) The prediction of PNPLA6 mutations using SIFT, Polyphen2, MutationTaster, MutationAssessor and CADD. (E) Conservative analysis of the glycine 1,129 and tryptophan 1,178-containing portions of the PNPLA6 protein. The glycine 1,129 and tryptophan 1,178 are highlighted. PNPLA6, patatin-like phospholipase domain containing 6; Crp, cAMP-binding domain of CRP.

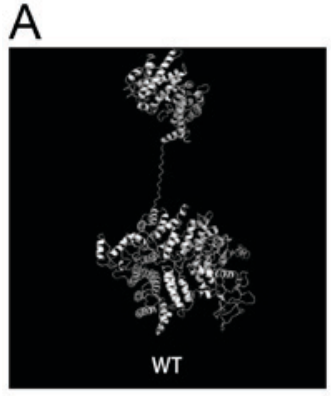

$\mathrm{B}$
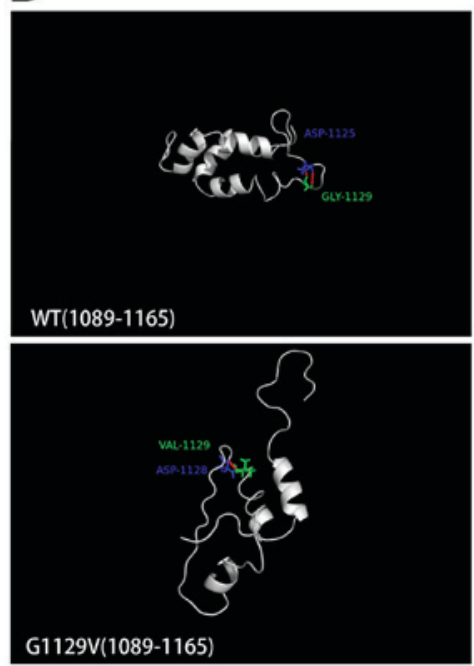
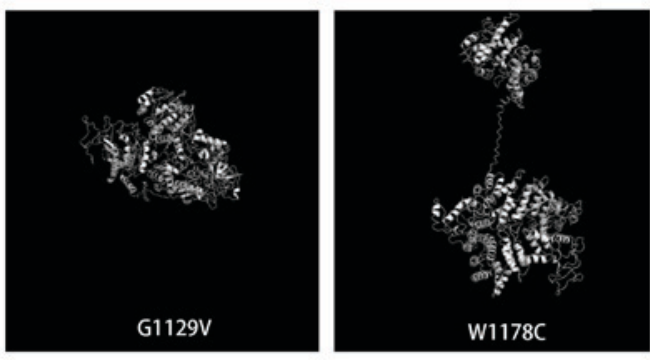

C

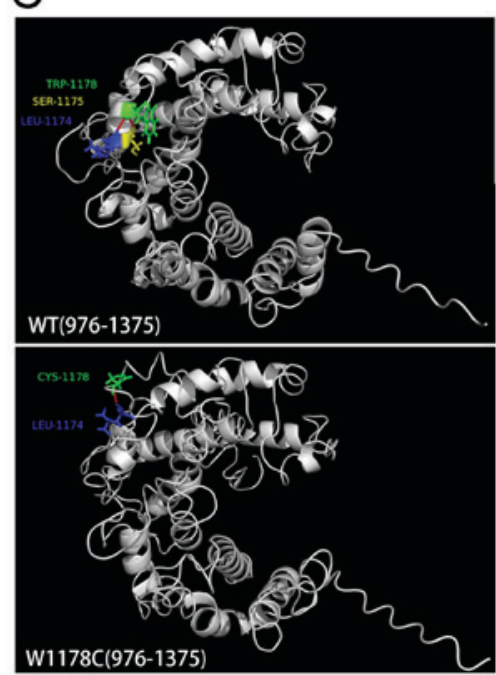

Figure 3. Structural modeling of WT and mutant (G1129V, W1178C) PNPLA6 proteins using I-TASSER. The models were visualized in PyMOL. (A) The three-dimensional structures of WT and the respective PNPLA6 mutant. (B) The structures of amino acids 1,089-1,165 from WT and G1129V PNPLA6. (C) The structures of amino acids 976-1,375 from WT and W1178C PNPLA6. Red line, hydrogen bond. WT, wild type PNPLA6, patatin-like phospholipase domain containing 6 . 


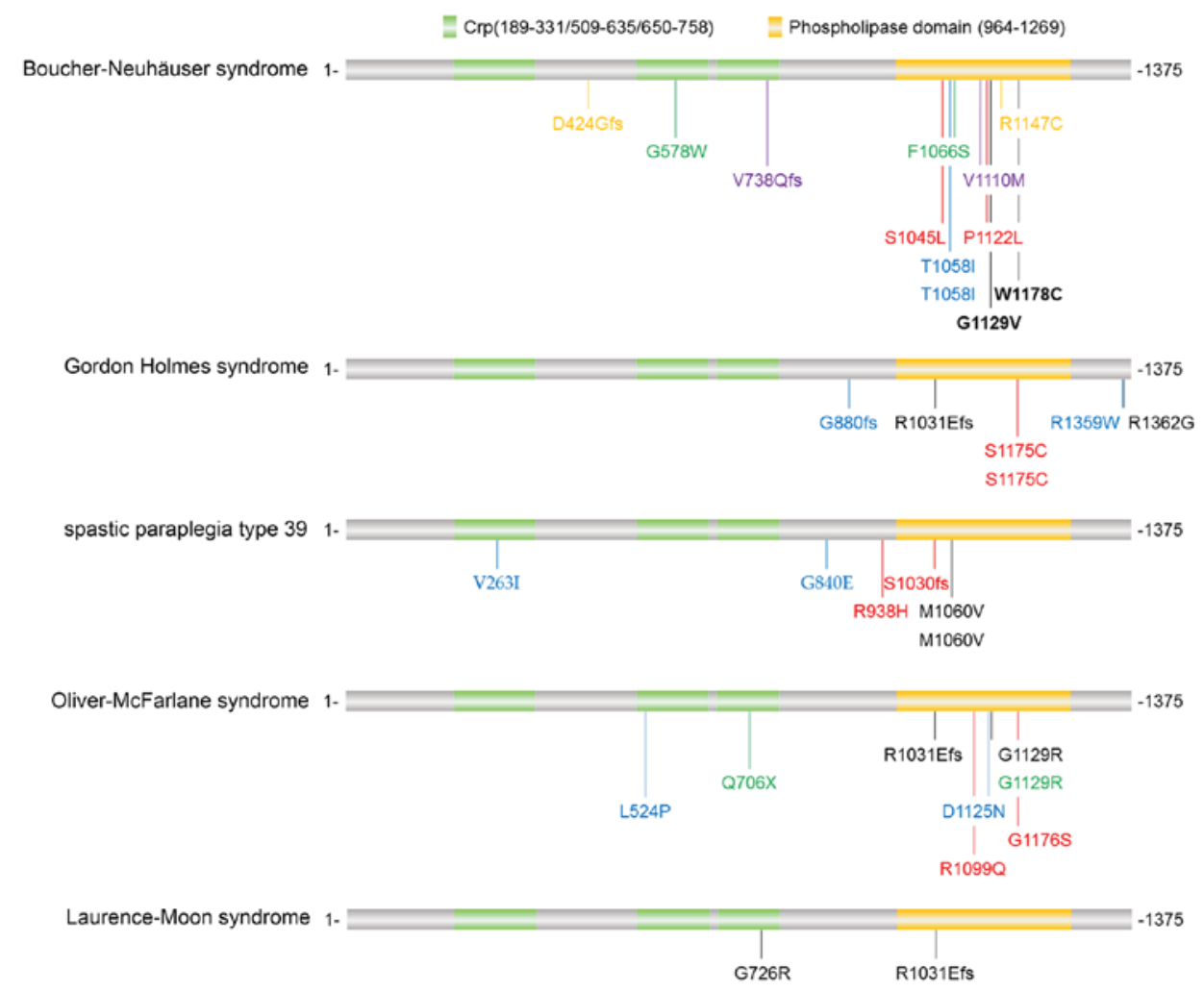

Figure 4. Mutations identified in patients with patatin-like phospholipase domain containing 6-related disorders. The biallelic variants from each patient are marked with the same color. Crp, cAMP-binding domain of CRP.

marked cerebellar atrophy, with widened cerebellar sulci and increased cerebrospinal fluid (Fig. 1C). Furthermore, MRI illustrated a reduction in the size the pituitary, the expansion of the right lateral side, and a reduction in the branches of the right middle cerebral artery (Fig. 1C and D). From these results, the proband was diagnosed as having BNS.

Whole exome sequencing. To understand the genetic mechanism, whole exome sequencing of the proband was performed. A total of 39.81 million clean reads were obtained following the removal of low-quality reads and adaptor or contaminant sequences. A total of $98.94 \%$ of the exome regions were covered. The sample had an average of 46.8 -fold coverage of the entire exon region, of which $93.9,80.6$ and $64.4 \%$ of the targeted regions were covered at least 10, 20 and 30 times, respectively. Using the GATK, a total of 21,797 coding SNVs and 584 small indels were identified. These included 10,054 missense variants, 73 nonsense variants, 189 splice site variants and 322 frame-shift indel mutations.

Compound heterozygous mutation in PNPLA6. A total of two PNPLA6 mutations (c.3386G >T, p.G1129V and c.3534G >C, p.W1178C; Fig. 2A and B) were identified. To determine whether the two PNPLA6 mutations (c.3386G>T, p.G1129V; c.3534G $>$ C, p.W1178C) were located in the same or different chromosomes, the mutations were analyzed in the parents of the proband. As exhibited in Fig. $2 \mathrm{~A}$ and $\mathrm{B}$, the proband inherited PNPLA6 (c.3534G $>$ C, p.W1178C) and PNPLA6 (c.3386G $>$ T, p.G1129V) from his father and mother, respectively. Therefore, the PNPLA6 mutations exist in a compound heterozygous form.
The PNPLA6 (c.3386G>T, p.G1129V) and PNPLA6 (c.3534G $>$ C, p.W1178C) mutations were located in the 29th and 30th exons, respectively. The two mutations were located in the phospholipid esterase domain of the PNPLA6 protein (Fig. 2C), and were predicted to be pathologically damaging using SIFT, Polyphen2, MutationTaster, MutationAssessor and CADD (Fig. 2D). Neither PNPLA6 (c.3386G>T, p.G1129V) nor PNPLA6 (c.3534G $>$ C, p.W1178C) were identified in the ExAC and 1,000 Genomes databases. Glycine 1,129 and tryptophan 1,178 are evolutionarily conserved (Fig. 2E). Structural modeling was additionally performed using I-TASSER (27). As exhibited in Fig. 3, mutations led to conformational alterations in the PNPLA6 protein. The overall conformation of W1178C PNPLA6 only demonstrated a minor alteration compared with wild-type (WT) PNPLA6, while the overall conformation of G1129V PNPLA6 was markedly altered (Fig. 3A). Glycine 1,129 formed a hydrogen bond with aspartic acid at 1,125 in WT PNPLA6; however, a G1129V mutation led to an alternative hydrogen bond between valine 1,129 and aspartic acid at 1,128 (Fig. 3B). Tryptophan 1,178 formed hydrogen bonds with serine 1,175 and leucine 1,174 in WT PNPLA6, whereas cysteine 1,178 only formed a hydrogen bond with leucine 1,174 in W1178C PNPLA6 (Fig. 3C).

PNPLA6 mutations have been identified in a panel of disorders, including Boucher-Neuhäuser syndrome, Gordon Holmes syndrome, spastic paraplegia type 39, Oliver-McFarlane syndrome and Laurence-Moon syndrome, with partly distinct and partly overlapping clinical phenotypes. For instance, Boucher-Neuhäuser syndrome and Gordon Holmes syndrome cause cerebellar ataxia and hypogonadotropic hypogonadism. However, Boucher-Neuhäuser syndrome additionally 
presents with chorioretinal dystrophy, whereas Gordon Holmes syndrome leads to brisk reflexes. By contrast, spastic paraplegia type 39 exhibits upper motor neuron involvement, peripheral neuropathy, and sometimes reduced cognitive functioning and/or cerebellar ataxia; Oliver-McFarlane syndrome exhibits trichomegaly, chorioretinal dystrophy, short stature, intellectual disability and hypopituitarism; and Laurence-Moon syndrome is characterized by chorioretinopathy, pituitary dysfunction, childhood onset of ataxia, spastic paraplegia and peripheral neuropathy $(16,28)$.

PNPLA6-associated disorders are usually diagnosed by clinical and neuroimaging results combined with biallelic pathogenic variants in PNPLA6. The mutations identified in patients with PNPLA6-associated disorders $(12,13,28-30)$ are illustrated in Fig. 4. The majority of the mutations were located in the phospholipid esterase domain of the PNPLA6 protein, although there was no disease-specific bias in these mutations. Hufnagel et al (28) envisioned that various PNPLA6 mutations may lead to different activity alterations in the neuropathy target esterase, and more severe reductions may cause more severe and early-onset presentations. However, further studies in animal models are required to uncover why mutations in one gene (PNPLA6) lead to various clinical presentations in different cell types.

In conclusion, the first BNS patient from China was identified in the present study. Although the proband did not exhibit gait ataxia at the age of 39 years old, MRI examination clearly indicated cerebellar atrophy. Genetic analysis confirmed the clinical manifestation. The present study implied that whole exome sequencing may be used for correct diagnosis and proper genetic counseling prior to the occurrence of obvious symptoms.

\section{Acknowledgements}

Not applicable.

\section{Funding}

The present study was financially supported by the National High-tech R\&D Program (grant no. 2015AA020502), National Basic Research Program of China (grant no. 2012CB517904), National Natural Science Foundation of China (grant nos. 31371187, 31501152, 31401218, 81770780 and 81728013), the Basic and Frontier Project from the Department of Science, Technology of Henan Province (grant no. 142300410071) and the Fundamental Research Funds for the Central Universities of Central South University (grant nos. 1053320170642, 1053320171214 and 1053320170804$)$.

\section{Availability of data and materials}

The datasets used and/or analyzed during the current study are available from the corresponding author on reasonable request.

\section{Authors' contributions}

RZ, YZ, WHL and JDL conceived the study. RZ, YW, JLL, ZLZ and DNC recruited the patients. YZ, JW and JDL performed the experiments. RZ, YZ, JW, YW, JLL, ZLZ, XTZ, DNC, WHL and JDL analyzed the data. YZ and JDL wrote the manuscript.

\section{Ethics approval and consent to participate}

Written informed consent was obtained. The present study was approved by the ethics committees of Central South University and the People's Hospital of Henan Province.

\section{Consent for publication}

Patients provided written informed consent for the use of data in this study.

\section{Competing interests}

The authors declare they have no competing interests.

\section{References}

1. Jbour AK, Mubaidin AF, Till M, El-Shanti H, Hadidi A and Ajlouni KM: Hypogonadotrophic hypogonadism, short stature, cerebellar ataxia, rod-cone retinal dystrophy, and hypersegmented neutrophils: A novel disorder or a new variant of Boucher-Neuhauser syndrome? J Med Genet 40: e2, 2003.

2. Kate MP, Kesavadas C, Nair M, Krishnan S, Soman M and Singh A: Late-onset Boucher-Neuhäuser Syndrome (late BNS) associated with white-matter changes: A report of two cases and review of literature. J Neurol Neurosurg Psychiatry 82: 888-891, 2011.

3. Lima-Martinez MM, Gil V, Zerpa J, Rivas P, Gomez-Perez R and Osuna J: Boucher-Neuhäuser syndrome. Endocrinol Nutr 60: 218-220, 2013.

4. Ling H, Unnwongse $\mathrm{K}$ and Bhidayasiri R: Complex movement disorders in a sporadic Boucher-Neuhäuser Syndrome: Phenotypic manifestations beyond the triad. Mov Disord 24: 2304-2306, 2009.

5. Tarnutzer AA, Gerth-Kahlert C, Timmann D, Chang DI, Harmuth F, Bauer P, Straumann D and Synofzik M: Boucher-Neuhäuser syndrome: Cerebellar degeneration, chorioretinal dystrophy and hypogonadotropic hypogonadism: Two novel cases and a review of 40 cases from the literature. J Neurol 262: 194-202, 2015.

6. Yu SI, Kim JL, Lee SG, Kim HW and Kim SJ: Ophthalmologic findings of Boucher-Neuhäuser syndrome. Korean J Ophthalmol 22: 263-267, 2008.

7. Santos AV, Saraiva PF and Breia PN: Significance of neuroimaging in the diagnosis of Boucher-Neuhauser syndrome. Acta Med Port 16: 193-195, 2003 (In Portuguese).

8. Makita Y: Ataxia-hypogonadism syndrome (Boucher-Neuhäuser syndrome). Ryoikibetsu Shokogun Shirizu: 238-239, 2001 (In Japanese).

9. Deik A, Johannes B, Rucker JC, Sánchez E, Brodie SE, Deegan E, Landy K, Kajiwara Y, Scelsa S, Saunders-Pullman R and Paisán-Ruiz C: Compound heterozygous PNPLA6 mutations cause Boucher-Neuhäuser syndrome with late-onset ataxia. J Neurol 261: 2411-2423, 2014.

10. Koh K, Kobayashi F, Miwa M, Shindo K, Isozaki E, Ishiura H, Tsuji S and Takiyama Y: Novel mutations in the PNPLA6 gene in Boucher-Neuhäuser syndrome. J Hum Genet 60: 217-220, 2015.

11. Matsuzaka Y, Ohkubo T, Kikuti YY, Mizutani A, Tsuda M, Aoyama Y, Kakuta K, Oka A, Inoko H, Sakabe K, et al: Association of sick building syndrome with neuropathy target esterase (NTE) activity in Japanese. Environ Toxicol 29: 1217-1226, 2014.

12. Synofzik M, Gonzalez MA, Lourenco CM, Coutelier M, Haack TB, Rebelo A, Hannequin D, Strom TM, Prokisch H, Kernstock C, et al: PNPLA6 mutations cause Boucher-Neuhauser and Gordon Holmes syndromes as part of a broad neurodegenerative spectrum. Brain 137: 69-77, 2014. 
13. Topaloglu AK, Lomniczi A, Kretzschmar D, Dissen GA, Kotan LD McArdle CA, Koc AF, Hamel BC, Guclu M, Papatya ED, et al: Loss-of-function mutations in PNPLA6 encoding neuropathy target esterase underlie pubertal failure and neurological deficits in Gordon Holmes syndrome. J Clin Endocrinol Metab 99: E2067-E2075, 2014

14. Richardson RJ, Hein ND, Wijeyesakere SJ, Fink JK and Makhaeva GF: Neuropathy target esterase (NTE): Overview and future. Chem Biol Interact 203: 238-244, 2013

15. Sogorb MA, Pamies D, Estevan C, Estévez J and Vilanova E: Roles of NTE protein and encoding gene in development and neurodevelopmental toxicity. Chem Biol Interact 259: 352-357, 2016.

16. Synofzik M, Hufnagel RB and Züchner S: PNPLA6-related disorders. In: GeneReviews ${ }^{\circledR}$ [Internet]. Pagon RA, Adam MP, Ardinger HH, et al (eds), University of Washington, Seattle, WA, 1993-2018.

17. Wiethoff S, Bettencourt C, Paudel R, Madon P, Liu YT, Hersheson J, Wadia N, Desai J and Houlden H: Pure cerebellar ataxia with homozygous mutations in the PNPLA6 gene. Cerebellum 16: 262-267, 2017.

18. Braslavsky D, Grinspon RP, Ballerini MG, Bedecarrás P Loreti N, Bastida G, Ropelato MG, Keselman A, Campo S, Rey RA and Bergadá I: Hypogonadotropic hypogonadism in infants with congenital hypopituitarism: A challenge to diagnose at an early stage. Horm Res Paediatr 84: 289-297, 2015.

19. Dwyer AA, Jayasena CN and Quinton R: Congenital hypogonadotropic hypogonadism: Implications of absent mini-puberty. Minerva Endocrinol 41: 188-195, 2016.

20. Kim SH: Congenital hypogonadotropic hypogonadism and kallmann syndrome: Past, present, and future. Endocrinol Metab (Seoul) 30: 456-466, 2015.

21. Manara R, Salvalaggio A, Favaro A, Palumbo V, Citton V, Elefante A, Brunetti A, Di Salle F, Bonanni G and Sinisi AA; Kallmann Syndrome Neuroradiological Study Group: Brain changes in Kallmann syndrome. AJNR Am J Neuroradiol 35: 1700-1706, 2014

22. Uchino A, Saito N, Mizukoshi W and Okada Y: Anomalous origin of the occipital artery diagnosed by magnetic resonance angiography. Neuroradiology 53: 853-857, 2011.
23. Li H and Durbin R: Fast and accurate short read alignment with Burrows-Wheeler transform. Bioinformatics 25: 1754-1760, 2009.

24. Wang K, Li M and Hakonarson H: ANNOVAR: Functional annotation of genetic variants from high-throughput sequencing data. Nucleic Acids Res 38: e164, 2010.

25. 1000 Genomes Project Consortium, Auton A, Brooks LD, Durbin RM, Garrison EP, Kang HM, Korbel JO, Marchini JL, McCarthy S, McVean GA and Abecasis GR: A global reference for human genetic variation. Nature 526: 68-74, 2015.

26. Kircher M, Witten DM, Jain P, O'Roak BJ, Cooper GM and Shendure J: A general framework for estimating the relative pathogenicity of human genetic variants. Nat Genet 46: 310-315, 2014.

27. Yang J, Yan R, Roy A, Xu D, Poisson J and Zhang Y: The I-TASSER suite: Protein structure and function prediction. Nat Methods 12: 7-8, 2015.

28. Hufnagel RB, Arno G, Hein ND, Hersheson J, Prasad M, Anderson Y, Krueger LA, Gregory LC, Stoetzel C, Jaworek TJ, et al: Neuropathy target esterase impairments cause Oliver-McFarlane and Laurence-Moon syndromes. J Med Genet 52: 85-94, 2015

29. Kmoch S, Majewski J, Ramamurthy V, Cao S, Fahiminiya S, Ren H, MacDonald IM, Lopez I, Sun V, Keser V, et al: Mutations in PNPLA6 are linked to photoreceptor degeneration and various forms of childhood blindness. Nat Commun 6 : $5614,2015$.

30. Rainier S, Bui M, Mark E, Thomas D, Tokarz D, Ming L, Delaney C, Richardson RJ, Albers JW, Matsunami N, et al: Neuropathy target esterase gene mutations cause motor neuron disease. Am J Hum Genet 82: 780-785, 2008.

(7) $\odot$ This work is licensed under a Creative Commons Attribution-NonCommercial-NoDerivatives 4.0 International (CC BY-NC-ND 4.0) License. 